\title{
Incontinentia pigmenti (Bloch-Sulzberger syndrome): seven case reports from one family
}

\author{
A SPALLONE \\ From the St Gerardo Hospital, Department of Ophthalmology, Monza (Milan), Italy
}

SUMMARY Seven members from a large family who showed signs of incontinentia pigmenti were examined. A clear $X$-linked dominant transmission was demonstrated, lethal in males. Study of this family shows that vascular abnormalities of the retina and disorders of the retinal pigment epithelium are the most important ocular lesions in the Bloch-Sulzberger syndrome.

Incontinentia pigmenti(Bloch-Sulzberger syndrome) is a genetic disease of the skin with generalised ectodermal and mesodermal dysplasia which may often involve the eyes ( $35 \%$ of the patients), ${ }^{\prime}$ hair, teeth, and central nervous system. Skin lesions are Correspondence to Dr A Spallone. usually present at birth or shortly after in the form of erythematous eruptions with linear vesiculations. The final stage is characterised by irregular macular streaks of brown to slate-grey pigmentation, distributed asymmetrically on the torso and sometimes on the limbs.

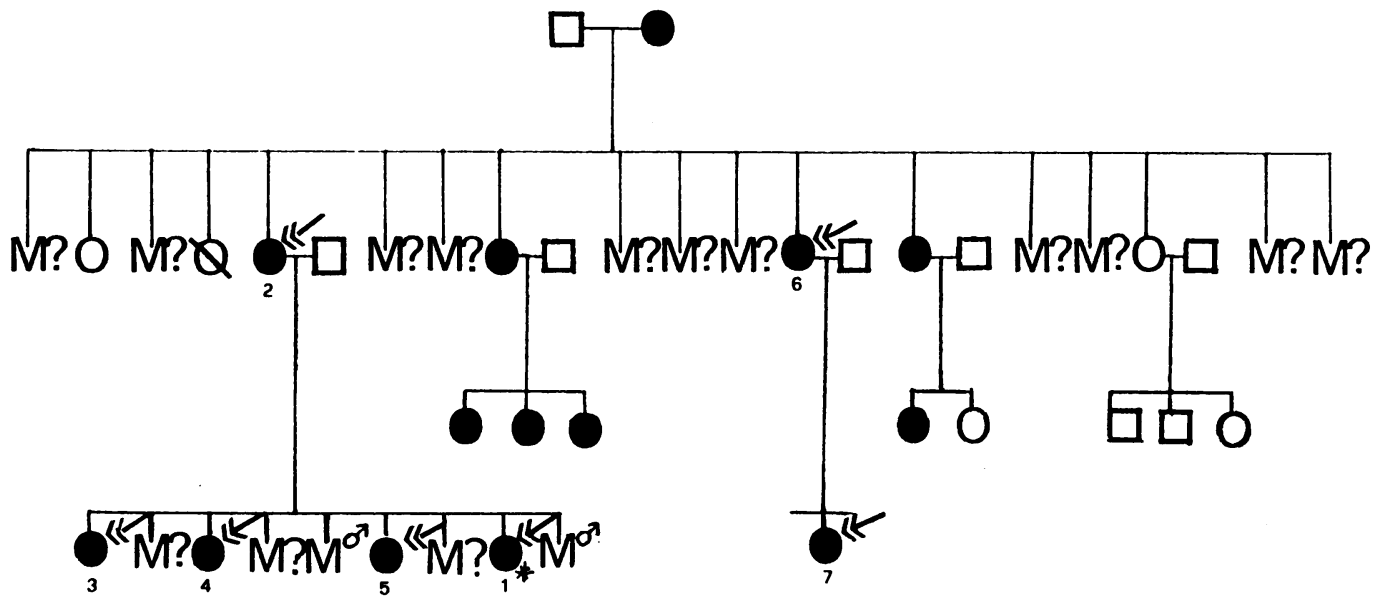

: Affected female

O: Not affected female

$\square:$ Not affected male Q:Dead for unknown cause
«:Case reports

*:Proband

M?.Miscarriages of unknown sex $\mathrm{M}^{0}$ :Miscarriages autoptically male

Fig. 1 Pedigree of family described. 


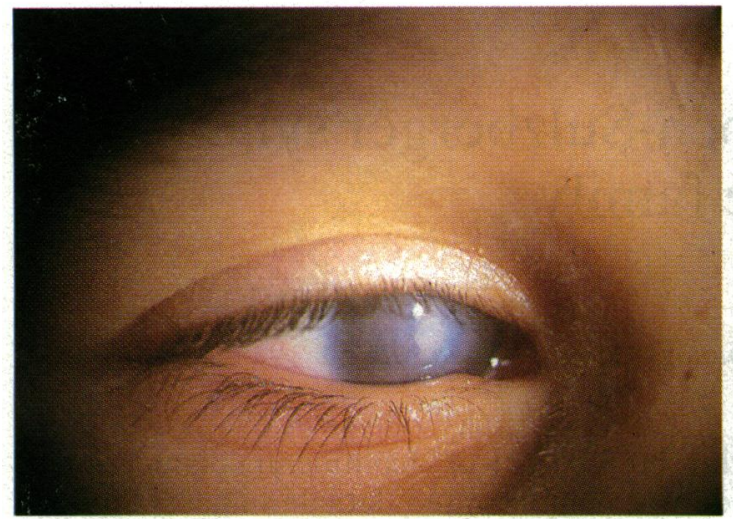

Fig. 2 Case 1. Right eye.

We have had the opportunity to study seven patients all of whom are members of one large family in which a total of 14 members were affected by incontinentia pigmenti. A clear $X$-linked dominant transmission was demonstrated, lethal in males (Fig. 1). The following seven case reports illustrate the most important ocular lesions of the disease.

\section{Case reports}

CASE 1

This was the proband. A female child, 4 years old, birth weight $3 \mathrm{~kg}$. The parents were not related. The dermatological symptoms of incontinentia pigmenti appeared immediately after birth.

Ocular examination showed nystagmus, oedematous cornea, and cataract (Fig. 2) in the right eye, microphthalmus with iridolenticular synechiae and complete cataract in the left eye. In this eye the iris was atrophic, with pigment irregularities (Fig. 3).

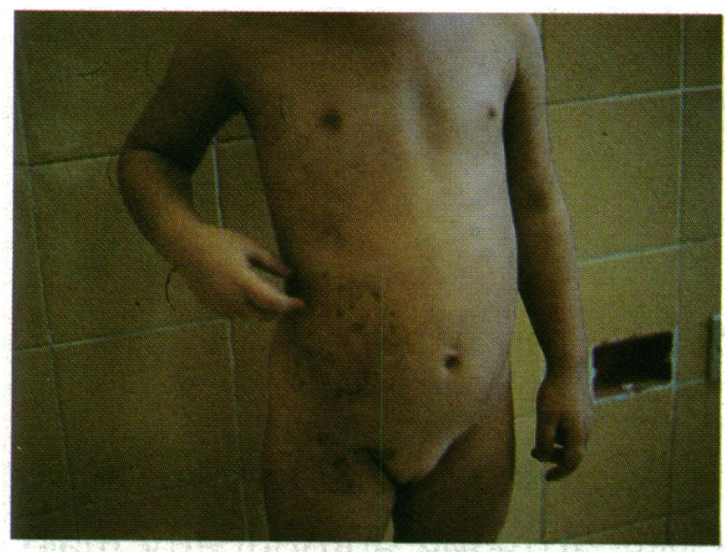

Fig. 4 Case 1. Classic whorls and streaks of pigment on torso and in right eye.

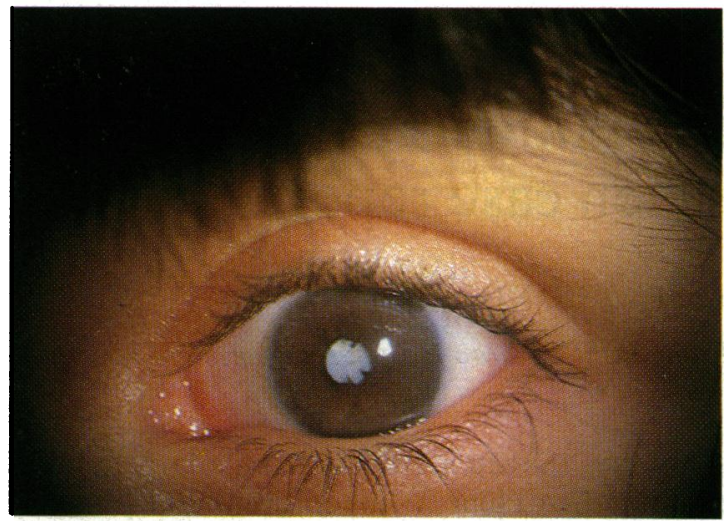

Fig. 3 Case 1. Left eye.

Visual acuity was no perception of light in both eyes. Ultrasonography revealed the presence of bilateral retinal detachment with dense membranes in the vitreous. A general medical examination showed the classic whorls and streaks of pigment on the torso and on the right leg (Fig. 4). The grandmother had had 18 pregnancies, 11 of which had been miscarriages. The seven fullterm pregnancies were all resulted in females. Five of these had ocular and dermatological problems; one died of an unknown cause, and the last one was healthy. The first of these five affected patients is the mother of our proband.

\section{CASE 2}

Mother of the proband, 37 years old, who has been affected by incontinentia pigmenti since birth.

Ocular examination showed intermittent exotropia. The visual acuity was 1.0 in both eyes. Both lenses were clear. In the ocular fundus in the right eye no anomalies could be found; by contrast in the left eye the retinal vessels were anomalous. Towards the temporal equator both venules and arterioles became tortuous, slightly kinked, of an irregular calibre, increased in number, and with preretinal fibrosis. Apart from this area the peripheral temporal part of the retina was completely avascular (Fig. 5). In the inferior nasal area there were small alterations of the retinal pigment epithelium. On general medical examination we noted some pigmentary alterations of the skin, the absence of the lateral upper incisors, and a conical appearance of the other teeth. The patient had had nine pregnancies; five of these had ended in miscarriages, two of which during necropsy were found to be males.

CASE 3

Sister of the proband, 14 years old. Immediately after birth the patient developed the dermatological signs of incontinentia pigmenti. 


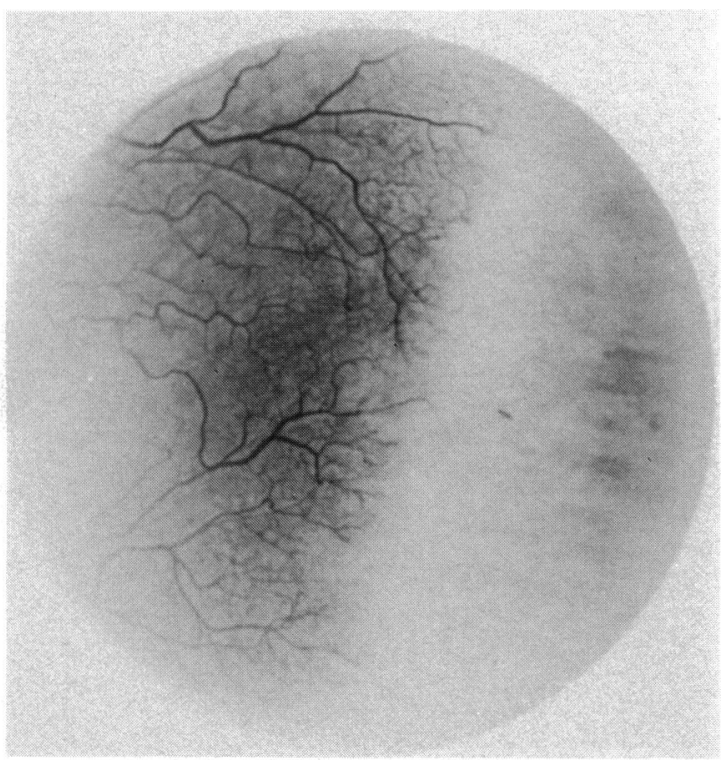

Fig. 5 Case 2. Fluorescein angiogram of left eye. In the temporal equator the retinal vessels are tortuous, slightly kinked, and increased in number. The peripheral temporal part of the retina is completely avascular.

Ophthalmological examination showed nystagmus and marked exotropia in the left eye. Visual acuity was 0.7 in the right eye and counting fingers in the left eye. The cornea and lens were both clear. The fundus in the right eye was normal. At the temporal equator

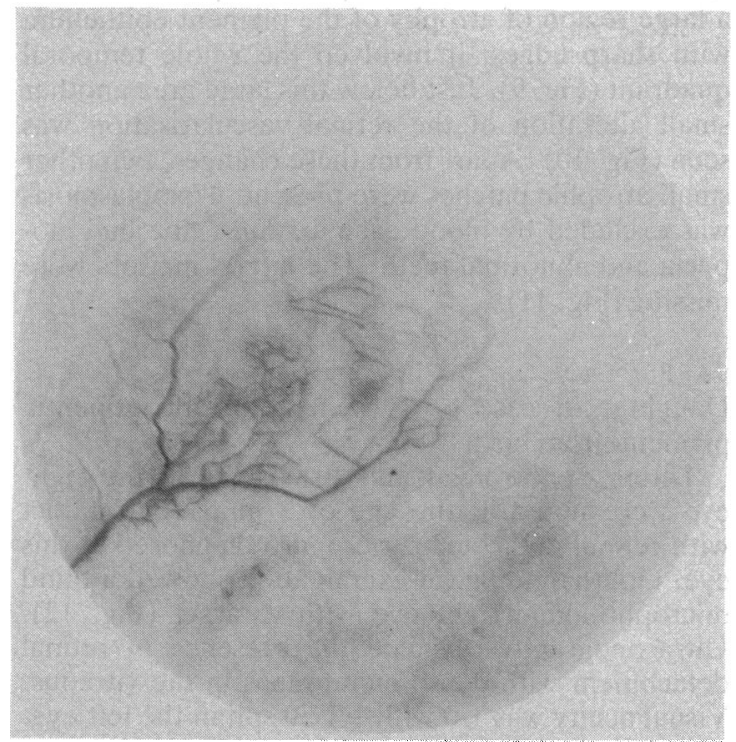

Fig. 6 Case 3. Fluorescein angiogram of the left eye, showing abnormal vascularisation of temporal peripheral retina.

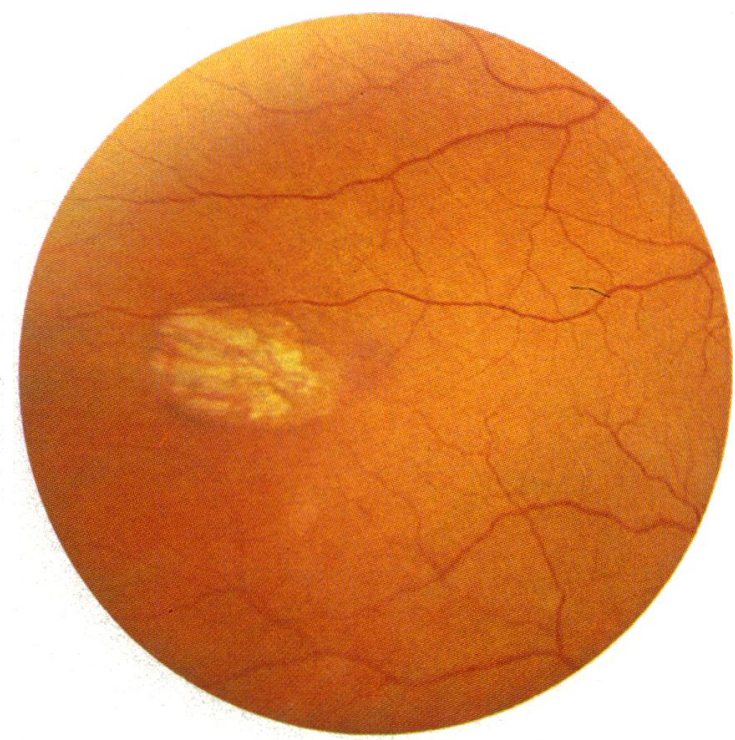

Fig. 7 Case 6. Fundus of right eye: alteration of pigment epithelium and choriocapillaris with sharp edges.

of the left eye the vessels arborised and connected in the form of an arteriovenous anastomosis, with preretinal fibrosis (Fig. 6). From this equatorial zone of abnormal vascularisation to the ora serrata the peripheral temporal part of the retina was avascular. In the sector inferior to the optic disc a small defect of the pigment epithelium was present.

This patient suffered from epilepsy and had spastic paralysis. In addition one half of the body was noted to be shorter than the other.

\section{CASE 4}

Sister of the proband, 10 years old. Incontinentia pigmenti from birth.

At the age of 3 months leucokoria was diagnosed in the right eye. This eye was enucleated because of suspected retinoblastoma. Visual acuity in the left eye as $0 \cdot 8$ with $-3 \mathrm{sph}$. The ocular fundus in the left eye showed vascular abnormalities in the equatorial retina very similar to that of case 2 . The peripheral temporal part of the retina was avascular. The typical pigmentary alterations of the skin were observed; dentition was incomplete, and some teeth were conical.

CASE 5

Sister of the proband, 8 years old, incontinentia pigmenti from birth. The anterior segment of the eyes was normal. Visual acuity was 0.6 in the right eye and 0.5 in the left eye. It was not possible to observe the ocular fundus in this patient. 


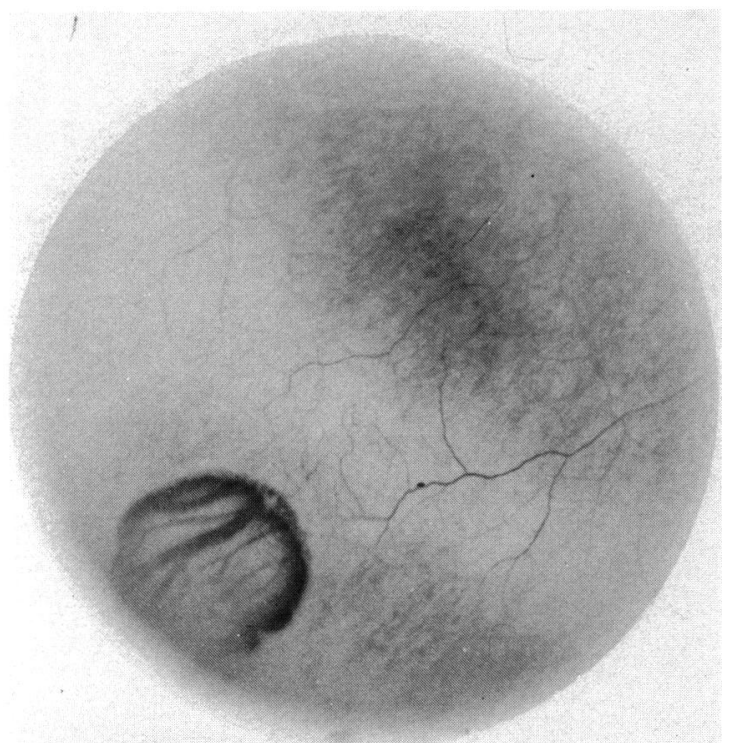

Fig. 8 Case 6. Fluorescein angiogram of right eye: in the temporal periphery a circular alteration of the pigment epithelium and choriocapillaris is present.

\section{CASE 6}

Aunt of the proband, 34 years old. Vesicular lesions and hyperpigmentation of the skin occurred two days after birth.

The right eye was exotropic. The visual acuity was 0.2 in the right eye with $-3.75 \mathrm{sph}$ and 0.8 in the left eye with $-8 \mathrm{sph}$. The corneas and lenses were both

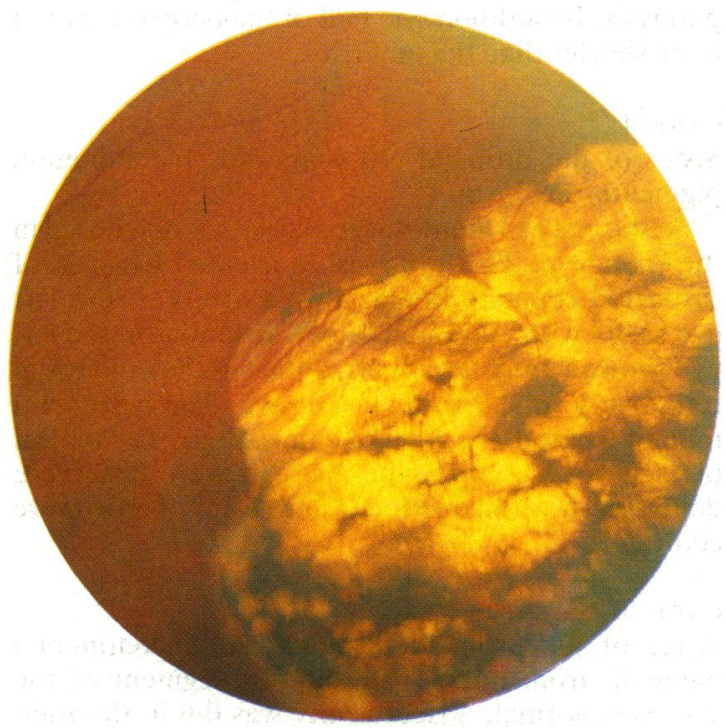

Fig. 9 Case 6. Fundus of left eye showing enormous atrophy of the pigment epithelium in the temporal quadrant.

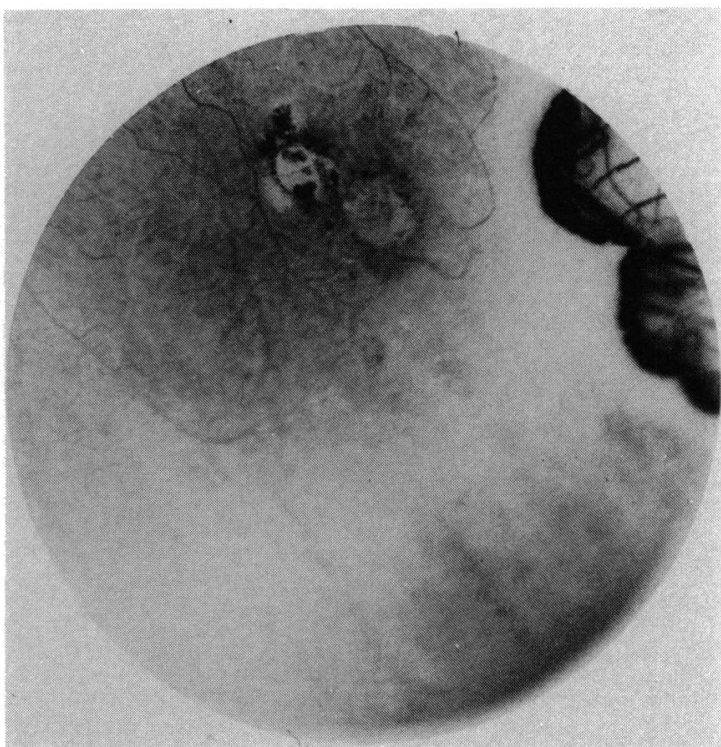

Fig. 10 Case 6. Fluorescein angiogram of left eye. Below the large atrophic area of the pigment epithelium are small alterations of the retinal vascularisation.

clear. Temporally to the macula a clearly defined change in the pigment epithelium and choriocapillaris with sharp edges was present (Fig. 7). In the temporal periphery another circular alteration of the pigment epithelium and choriocapillaris was visible (Fig. 8). This zone also showed mild anomalies of the retinal vascularisation. In the left eye it was possible to see a large region of atrophy of the pigment epithelium with sharp edges; it involved the whole temporal quadrant (Fig. 9). Just below this large area another small alteration of the retinal vascularisation was seen (Fig. 10). Apart from these changes, two other small atrophic patches were present. Toxoplasmosis was excluded by blood examinations. She had alopecia and abnormal teeth. The lateral incisors were missing (Fig. 11).

CASE 7

Daughter of case 6,11 years old. Incontinentia pigmenti from birth.

Tilting of the head and strabismus in the right eye were noted at the age of 3 months. Cataract with retinal detachment were also diagnosed in this eye. Ophthalmological examination showed a blind microphthalmic right eye with cataract (Fig. 12). Ultrasonography revealed the presence of retinal detachment with dense membranes in the vitreous. Visual acuity was 1.0 with $-1.50 \mathrm{sph}$ in the left eye. In this eye the cornea and lens were both clear. The ocular fundus showed only a small patch of pigment epithelium atrophy without alteration of the 


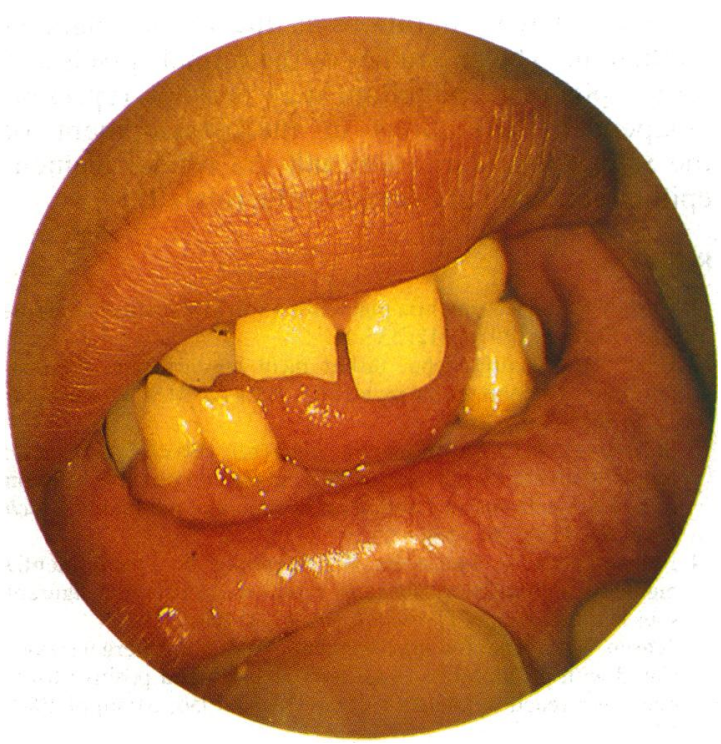

Fig. 11 Case 6. Abnormal configuration of the teeth.

retinal circulation (Fig. 13). She had marked dental anomalies, alopecia, and the typical streaks of pigment on the torso.

\section{Discussion}

The term incontinentia pigmenti was first used by Bloch $^{2}$ in 1926, when during a histological examination he observed abnormalities of the pigment cells of the epithelium of the skin, which were thought to be 'incontinent' of melanin. In 1938 Sulzberger $^{3}$ found other ectodermal defects in association with this condition. In 1954 Franceshetti and Jadassohn ${ }^{4}$ divided the disorder into two types: the classic incontinentia pigmenti or Bloch-Sulzberger variety,

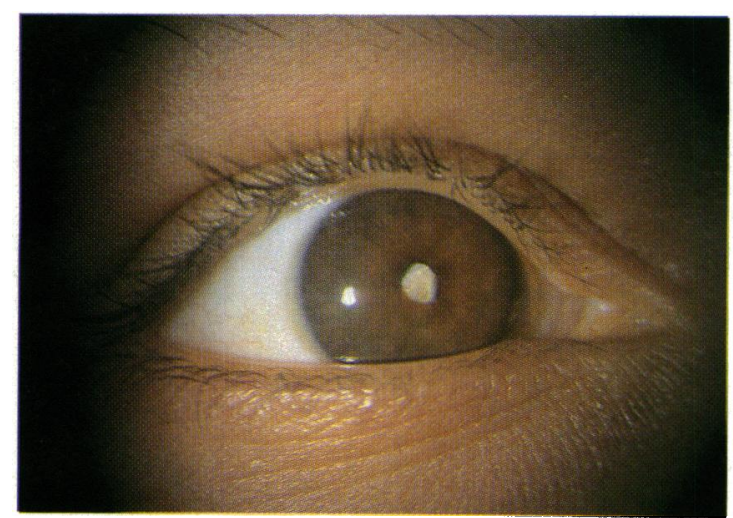

Fig. 12 Case 7. Microphthalmic right eye with complicated cataract.

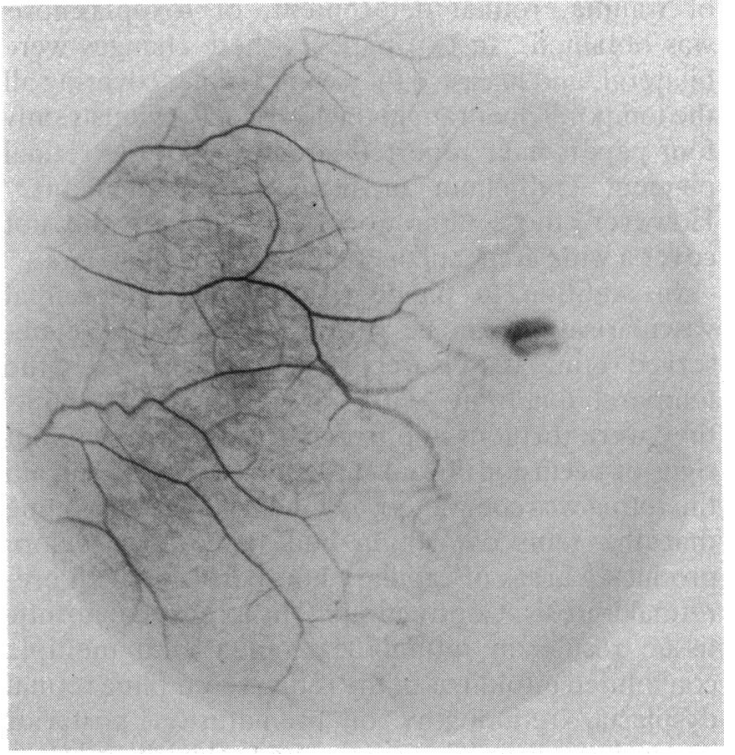

Fig. 13 Case 7. Fluorescein angiogram of left eye: small patch of pigment epithelium atrophy in the peripheral retina is present.

which occurs almost exclusively in females, and the Naegli type (or reticular) with a dominant mode of transmission, which involves both sexes without ocular malformations.

Our family clearly belongs to the Bloch-Sulzberger variety, and it is certainly the largest family ever described. Ocular abnormalities are generally found in $35 \%$ of the cases, and they probably represent the most severe systemic anomalies associated with incontinentia pigmenti. Our patients confirm this last point, and they illustrate the whole range of ocular lesions. One patient (case 4) underwent enucleation at 6 months of age because of suspected retinoblastoma. Our proband was completely blind. Myopia was present in three patients, strabismus with amblyopia in five, and cataract in three. It is interesting to note that cataracts were present only in the cases with retinal detachment, and they were probably caused by the retinal detachment. Microphthalmus was present in three cases, nystagmus in two. Ophthalmoscopically it was possible to see retinal abnormalities in four patients. In two other cases a dense cataract did not permit us to observe the fundus, but ultrasonography showed abnormalities of the vitreous, retinal detachment, and thickening of the choroid.

In three of our cases, characteristic and well marked changes in the retinal pigment epithelium and choriocapillaris were visible during ophthalmoscopy as of large plaques, which are probably analogous to those observed on the skin. No history 
of trauma, retinal detachment, or toxoplasmosis was obtained. ${ }^{5}$ In two patients these changes were bilateral, and in case 6 they were visible, covering all the temporal quadrant in the left eye. Previously only four papers have reported alterations of the retinal pigment epithelium in incontinentia pigmenti. ${ }^{6.9}$ However, those alterations were limited, did not cover a wide area, and were less serious than ours.

In addition to pigment epithelium the retinal vascularisation can be seriously affected. We observed retinal vessels were sharply interrupted in the temporal quadrant, with an arteriovenous shunt; they were tortuous and irregular in calibre, without signs of occlusion. From this zone to the ora serrata the retina was completely avascular. One can assume that the temporal retina had failed to develop, producing areas of capillary non-perfusion with preretinal fibrosis. Contraction of this preretinal fibrotic tissue results in retinal detachment and multiple convoluted infoldings of the retina resembling retinal dysplasia, retinopathy of prematurity, posterior hyperplastic primary vitreous, and other disorders.

The retinal vascular abnormalities and the disorders of pigment epithelium are the most important ocular lesions in incontinentia pigmenti. Some authors ${ }^{810}$ have inferred a relation between retinal pigment epithelium anomalies and fibrovascular changes. However, our findings showed that this relationship is not so clear. In fact in case 6 there was a noticeable alteration of the retinal pigment epithelium and few vascular defects. By contrast in cases 2 and 3 we noted serious defects of the retinal vessels and few alterations in the retinal pigment epithelium. The anomalies of the retinal vessels and retinal pigment epithelium may perhaps represent independent alterations in the development of the retinal vascularisation and the retinal pigment epithelium, and not necessarily be related.

\section{References}

1 Carney RG. Incontinentia pigmenti: a world statistical analysis. Arch Dermatol 1976; 112: 535-42.

2 Bloch B. Eigentümilche, bisher nicht beschriebene Pigmentaffektion (incontinentia pigmenti). Schweiz Med Wochenschr 1926; 56: 404-5.

3 Sulzberger MB. Incontinentia pigmenti (Bloch-Sulzberger); report of an additional case, with comment on possible relation to a new syndrome of familial and congenital anomalies. Arch Dermatol 1938; 38: 57-9.

4 Franceschetti A, Jadassohn W. A propos de l'incontinentia pigmenti, délimitation de deux syndromes differénts figurant sous le même terme. Dermatologica 1954; 108: 1-28.

5 Jensen VA. Incontinentia pigmenti (Bloch-Sulzberger) associated with proliferative eyeground changes and positive toxoplasmosis reaction. Acta Psychiatr Scand 1956; 31(suppl 108): 197.

6 Fischbein FI, Schub M, Lesko WS. Incontinentia pigmenti, pheochromocytoma and ocular abnormalities. Am J Ophthalmol 1972; 73: 961-4.

7 McCrary JA, Smith JL. Conjuntival and retinal incontinentia pigmenti. Arch Ophthalmol 1968; 79: 417-22.

8 Rosenfeld SI, Smith ME. Ocular findings in incontinentia pigmenti. Ophthalmology 1985; 92: 543-6.

9 Rabb EL. Ocular lesions in incontinentia pigmenti. $J$ Pediatr Ophthalmol Strabismus 1983; 420: 42-8.

10 Mensheha-Manhart O, Rodriguez MM, Shields JA, Shannon GM, Mirabelli RP. Retinal pigment epithelium in incontinentia pigmenti. Am J Ophthalmol 1975; 79: 571.

Accepted for publication 11 August 1986. 